\title{
Crossings in grid drawings
}

\author{
Vida Dujmović \\ School of Electrical Engineering and Computer Science \\ University of Ottawa \\ Ottawa, Canada \\ vdujmovi@uottawa.ca
}

\author{
Pat Morin \\ School of Computer Science \\ Carleton University \\ Ottawa, Canada \\ morin@scs.carleton.ca
}

\section{Adam Sheffer}

The Blavatnik School of Computer Science

Tel Aviv University

Tel Aviv, Israel

sheffera@post.tau.ac.il

\begin{abstract}
Submitted: Jan 3, 2013; Accepted: Feb 20, 2014; Published: Feb 28, 2014
Mathematics Subject Classification: 05C10
\end{abstract}

\begin{abstract}
We prove tight crossing number inequalities for geometric graphs whose vertex sets are taken from a $d$-dimensional grid of volume $N$ and give applications of these inequalities to counting the number of crossing-free geometric graphs that can be drawn on such grids.

In particular, we show that any geometric graph with $m \geqslant 8 N$ edges and with vertices on a 3D integer grid of volume $N$, has $\Omega\left(\left(m^{2} / N\right) \log (m / N)\right)$ crossings. In $d$ dimensions, with $d \geqslant 4$, this bound becomes $\Omega\left(m^{2} / N\right)$. We provide matching upper bounds for all $d$. Finally, for $d \geqslant 4$ the upper bound implies that the maximum number of crossing-free geometric graphs with vertices on some $d$-dimensional grid of volume $N$ is $N^{\Theta(N)}$. In 3 dimensions it remains open to improve the trivial bounds, namely, the $2^{\Omega(N)}$ lower bound and the $N^{O(N)}$ upper bound.
\end{abstract}

\section{Introduction}

The study of crossings in drawings of graphs has a long history. Euler's Formula states that the maximum number of edges in an $n$ vertex planar graph - one that can be drawn in the plane without crossings - is $3 n-6$. Using Euler's Formula and careful counting, Ajtai et al. [3] showed that any plane drawing of a graph with $n$ vertices and $m \geqslant 4 n$ edges has at least $\mathrm{cm}^{3} / \mathrm{n}^{2}$ crossing pairs of edges, for some constant $c \geqslant 1 / 100$. The same authors used this to prove their main result: The maximum number of non-crossing subgraphs of any graph drawn on any fixed set of $n$ points is $2^{O(n)}$. 
The lower bound, $\mathrm{cm}^{3} / \mathrm{n}^{2}$, on the number of crossings in a plane drawing has since become known as "the Crossing Lemma" or "the Crossing Number Inequality" and has subsequently found many other applications. Székely [29] showed that this inequality can be used to give very simple proofs of many results in incidence geometry, including a proof of the Szemerédi-Trotter Theorem on point-line incidences [30]. Székely's method has since been used for many combinatorial geometry problems; the most famous of these applications is probably the result of Dey [10] on the maximum number $k$-sets of a point set.

Ajtai et al.'s proof of the Crossing Lemma works by summing the number of crossings in two different ways. More recently, a proof "from the book" that uses the probabilistic method to obtain a better constant, $c \geqslant 1 / 64$, was discovered by Chazelle, Sharir, and Welzl (See Aigner and Ziegler [2, Chapter 30, Theorem 4]). By pushing the "from the book" proof even further, Pach et al. [22] improved the constant $c$ to $c \geqslant 1024 / 31827 \approx$ $1 / 33.1$. Very recently, Ackerman [1], has improved the constant even further to $c \geqslant 1 / 29$.

The main result of Ajtai et al.- that the maximum number of crossing-free graphs that can be drawn on any point set of size $n$ is $2^{O(n)}$ - has also been the starting point for many research problems. The original bound, which was $O\left(10^{13 n}\right)$, has been improved repeatedly to the current record of $O\left(187.53^{n}\right)$ [25]. The result has also been tightened for special classes of crossing-free graphs including triangulations $\left(O\left(30^{n}\right)\right)$ [24], spanning cycles $\left(O\left(54.55^{n}\right)\right)$ [26], perfect matchings $\left(O\left(10.05^{n}\right)\right)$ [27], spanning trees $\left(O\left(141.07^{n}\right)\right)$ [18], and forests $\left(O\left(160.55^{n}\right)\right)[18,24]$. A webpage containing an up-to-date compendium of these types of results is maintained by the third author [28].

\section{$1.1 \quad$ Geometric Grid Graphs}

The goal of the present paper is to extend the results of Ajtai et al. to graph drawings in higher dimensions. In particular, we extend their results to graphs drawn on grids. For any positive integers $X_{1}, \ldots, X_{d}$, the $d$-dimensional $X_{1} \times \cdots \times X_{d}$ grid is a finite subset of the $d$-dimensional natural lattice, $\mathbb{N}^{d}$, given by

$$
\mathbb{N}\left(X_{1}, \ldots, X_{d}\right)=\left\{\left(x_{1}, \ldots, x_{d}\right): x_{i} \in\left\{1, \ldots, X_{i}\right\} \text { for all } i \in\{1, \ldots, d\}\right\} .
$$

The volume of the $X_{1} \times \cdots \times X_{d}$ grid is $\prod_{i=1}^{d} X_{i}$, i.e,. the number of points in the grid.

A ( $d$-D) geometric (grid) graph, $G$, is a graph with vertex set $V(G) \subseteq \mathbb{N}^{d}$. Throughout this paper, for two vertices $u$ and $w$ in a geometric graph, $G$, we will use the notation $u w$ to refer both to the open line segment with endpoints $u$ and $w$ and to the edge $u w \in E(G)$, if present. The volume, $\operatorname{vol}(G)$, of $G$ is the volume of the minimal $X_{1} \times \cdots \times X_{d}$ grid that contains $V(G)$.

A geometric grid graph, $G$, is proper if, for every edge $u w \in E(G)$, and every vertex $x \in V(G)$, we have that $x \notin u w$. That is, $G$ is proper if no edge passes through a vertex. For the remainder of this paper, the phrase "geometric grid graph" and "grid graph" should be interpreted as "proper geometric grid graph."

Two edges $u w$ and $x y$ in a geometric grid graph cross if they have a point in common. When this happens, we say that $u w$ and $x y$ form a crossing. We define $\operatorname{cr}(G)$ as the 
number of crossings in $G .{ }^{1}$ To avoid later confusion, the reader should note that $\operatorname{cr}(G)$ counts pairs of crossing edges, so that if $k$ edges meeting in a common point, then this contributes $\left(\begin{array}{l}k \\ 2\end{array}\right)$ to the value of $\operatorname{cr}(G)$. We say that $G$ is crossing-free if $\operatorname{cr}(G)=0$. Finally, we define

$$
\operatorname{cr}_{d}(N, m)=\min \{\operatorname{cr}(G): G \text { is a } d \text {-D grid graph, }|E(G)|=m \text {, and } \operatorname{vol}(G) \leqslant N\} .
$$

That is, $\mathrm{cr}_{d}(N, m)$ is the minimum number of crossings in any $d$-D geometric grid graph with $m$ edges and volume no more than $N$.

We are also interested in the maximum number, $\operatorname{ncs}_{d}(N)$, of crossing-free $d$-D geometric grid graphs that can be drawn on any particular grid of volume at most $N$. That is,

$$
\operatorname{ncs}_{d}(N)=\max \left\{\mid\left\{G: V(G) \subseteq \mathbb{N}\left(X_{1}, \ldots, X_{d}\right) \text { and } \operatorname{cr}(G)=0\right\} \mid: \prod_{i=1}^{d} X_{i} \leqslant N\right\} .
$$

Results on plane drawings of graphs have immediate implications for $\mathrm{cr}_{2}(N, m)$ and $\operatorname{ncs}_{2}(N)$ :

1. Euler's Formula implies that $\mathrm{cr}_{2}(N, 3 N-5) \geqslant 1$,

2. Ajtai et al.'s Crossing Lemma implies that $\mathrm{cr}_{2}(N, m) \geqslant \mathrm{cm}^{3} / N^{2}$ for $m \geqslant 4 N$, and

3. Ajtai et al.'s upper-bound of $2^{O(n)}$ on the number of planar graphs that can be drawn on any planar point set of size $n$ implies that $\operatorname{ncs}_{2}(N) \in 2^{O(N)}$.

Bose et al. [5] show that the maximum number of edges in a crossing-free $d$-D geometric grid graph of volume $N$ is at most $\left(2^{d}-1\right) N-\Theta\left(N^{(d-1) / d}\right)$. This result is analogous to Euler's Formula in the sense that it shows that such graphs have a linear number of edges. It also implies, for example, that $\mathrm{cr}_{d}\left(N,\left(2^{d}-1\right) N\right) \geqslant 1$. Since Euler's Formula is the main property of planar graphs used by Ajtai et al. to prove their results, it seems reasonable that bounds similar to those of Ajtai et al. should hold for $d$-D geometric grid graphs.

The key difference, however, is that unlike Euler's formula, the bound of Bose et al. depends on the volume, $N$, of the grid and not on the number, $n$, of vertices in the graph. For $d \geqslant 3$ it is not possible to obtain non-trivial bounds on the number of crossings that depend only on the number of edges and vertices. This is because every graph on $n=N^{1 / 3}$ vertices can be drawn as a 3-D geometric grid graph of volume $N$ [9].

\section{$1.2 \quad$ New Results}

In this paper, we study $\operatorname{cr}_{d}(N, m)$ and $\operatorname{ncs}_{d}(N)$ for $d \geqslant 3$ and prove the results shown in Table 1. In Table 1, and throughout this paper, we assume that $d$ is a constant that is independent of $N$ and $m$, so that the $O, o, \Omega, \omega$, and $\Theta$ notations hide factors that depend only on $d$.

\footnotetext{
${ }^{1}$ Note that this is different from the planar crossing number, usually also denoted $\operatorname{cr}(G)$, that is the minimum number of crossings in any drawing of the (non-geometric) graph $G$. Our definition is more akin to the crossing number of a drawing of a graph.
} 


\begin{tabular}{r|lll}
$d$ & $\operatorname{cr}_{d}(N, m)$ & $\operatorname{ncs}_{d}(N)$ & References \\
\hline 2 & $\Theta\left(m^{3} / N^{2}\right)$ & $2^{\Theta(N)}$ & {$[3]$} \\
3 & $\Theta\left(\left(m^{2} / N\right) \log (m / N)\right)$ & & Theorems 1 and 2 \\
$\geqslant 4$ & $\Theta\left(m^{2} / N\right)$ & $2^{\Theta(N \log N)}$ & Theorems 3, 4, and 5
\end{tabular}

Table 1: Old and new results on crossings in $d$-D geometric grid graphs.

Our results show that the situation in three and higher dimensions is significantly different than in two dimensions. For all $d \geqslant 4$, and $m \geqslant 2^{d} N, \mathrm{cr}_{d}(N, m) \in \Theta\left(m^{2} / N\right)$ and even $\mathrm{cr}_{3}(N, m)$ is only $\Theta\left(\left(m^{2} / N\right) \log (m / N)\right)$. There are therefore geometric grid graphs with $\Omega\left(N^{2}\right)$ edges that have only $O\left(N^{3}\right)$ crossings $\left(O\left(N^{3} \log N\right)\right.$ crossings in 3-d). In contrast, in 2 dimensions, any graph with $n$ vertices and $\Omega\left(n^{2}\right)$ edges has $\Omega\left(n^{4}\right)$ crossings.

For $d \geqslant 4$, the bounds on $\operatorname{cr}_{d}(N, m)$ are strong enough to show that $\operatorname{ncs}_{d}(N) \in$ $2^{\Theta(N \log N)}$. Thus, the number, $2^{\Theta(N \log N)}$, of crossing-free graphs whose vertex set comes from a specific $d$-dimensional grid having $N$ points is much larger than the number, $2^{\Theta(N)}$, of crossing-free graphs that can be drawn on any planar point set of size $N$.

\subsection{Related Work}

The study of crossing-free 3-D geometric grid graphs is an active area in the field of graph drawing. A $d$-D grid drawing of a graph, $G$, is a one-to-one mapping $\varphi: V(G) \rightarrow \mathbb{N}^{d}$. Any drawing, $\varphi$, yields a geometric grid graph, $\varphi(G)$, with vertex set $V(\varphi(G))=\{\varphi(u): u \in$ $V\}$ and edge set $E(\varphi(G))=\{\varphi(u) \varphi(w): u w \in E(G)\}$. The drawing $\varphi$ is crossing-free if the geometric grid graph $\varphi(G)$ is crossing-free and the volume of $\varphi$ is the volume of $\varphi(G)$.

Cohen et al. [9] showed that the complete graph on $n$ vertices, and therefore any graph on $n$ vertices, has a crossing-free 3-D grid drawing of volume $O\left(n^{3}\right)$ and this is optimal. However, for many classes of graphs, sub-cubic volume 3-D grid drawings are possible; this includes sufficiently sparse graphs $\left(O\left(m^{4 / 3} n\right)\right)$ [14], graphs with maximum degree $\Delta$ and other $\Delta$-degenerate graphs $\left(O(\Delta m n), O\left(\Delta^{15 / 2} m^{1 / 2} n\right)\right)$ [14, 15], $\chi$-colorable graphs $\left(O\left(\chi^{2} n^{2}\right), O\left(\chi^{6} m^{2 / 3} n\right)\right)[23,14]$, graphs taken from some proper minor-closed family of graphs $\left(O\left(n^{3 / 2}\right)\right)$ [14], planar graphs $\left(O\left(n \log ^{16} n\right)\right)$ [11], outerplanar graphs $(O(n))$ [16], and graphs of constant treewidth $(O(n))$ [12].

The work most closely related to the current work, in that it presents an extremal result relating crossings, volume, and number of edges, is that of Bose et al. [5], who showed that the maximum number of edges in a crossing-free $d$-D geometric grid graph, $G$, with vertex set $V(G) \subseteq \mathbb{N}\left(X_{1}, \ldots, X_{d}\right)$, is exactly

$$
\prod_{i=1}^{d}\left(2 X_{i}-1\right)-\prod_{i=1}^{d} X_{i}
$$

For a fixed volume, $N=\prod_{i=1}^{d} X_{i}$, maximizing (1) gives $X_{1}=\cdots=X_{d}=N^{1 / d}$, in which case (1) becomes $\left(2^{d}-1\right) N-\Theta\left(N^{(d-1) / d}\right) \leqslant\left(2^{d}-1\right) N$. We state this here as lemma since 
we make use of it several times.

Lemma 1 (Bose et al. 2004). In any crossing-free d-D geometric grid graph, G, of volume $N$, we have that $|E(G)| \leqslant\left(2^{d}-1\right) N$.

Lemma 1 immediately yields the upper-bound $\operatorname{ncs}_{d}(N) \in 2^{O(N \log N)}$ (see the beginning of Section 4). It also yields the lower-bound $\mathrm{cr}_{d}(m) \geqslant m-\left(2^{d}-1\right) N$ since, if a geometric grid graph $G$ has $m \geqslant 2^{d-1} N$ edges, then we can remove an edge from $G$ that eliminates at least one crossing. Since this can be repeated until $G$ has $m \leqslant 2^{d-1} N$ edges, this implies that $G$ has at least $m-\left(2^{d}-1\right) N$ crossings.

Finally, we note that Bukh and Hubard [7] present a different definition of crossing number for 3-dimensional geometric graphs that are not necessarily grid graphs. In their definition, a 4-tuple of vertex-disjoint edges forms a space crossing if there is a line that intersects every edge in the 4-tuple. The space crossing number, $\mathrm{cr}_{4}(G)$, of a 3 -d geometric graph, $G$, is the number of space crossings formed by $G$ 's edges. They show that a 3-d geometric graph $G$ with $n$ vertices and $m \geqslant 4^{41} n$ edges has a space crossing number $\mathrm{cr}_{4}(G) \in \Omega\left(m^{6} /\left(n^{4} \log ^{2} n\right)\right)$. An easy lifting argument shows that this bound on the space crossing number almost implies the Crossing Lemma; specifically, it shows that the number of crossings in a graph with $n$ vertices and $m$ edges drawn in the plane is $\Omega\left(m^{3} /\left(n^{2} \log n\right)\right)$.

\section{3-Dimensional Geometric Grid Graphs}

In this section, we present upper and lower bounds on $\operatorname{cr}_{3}(N, m)$. Here, and in the remainder of the paper we use the notation $u_{i}, i \in\{1, \ldots, d\}$, to denote the $i$ th coordinate of the $d$-dimensional point $u$. Thus, for a point $u \in \mathbb{R}^{3}, u_{1}, u_{2}$, and $u_{3}$ are $u^{\prime}$ 's $\mathrm{x}_{-}, \mathrm{y}-$, and z-coordinates, respectively.

\subsection{The Lower Bound}

Theorem 1. For all $m \geqslant 8 N, \operatorname{cr}_{3}(N, m) \in \Omega\left(\left(m^{2} / N\right) \log (m / N)\right)$.

Proof. Let $G$ be any geometric grid graph with $V(G) \subseteq \mathbb{N}(X, Y, Z)$, with $X Y Z \leqslant N$, and $|E(G)|=m$. (That is, $G$ is a $3-\mathrm{D}$ geometric grid graph with $m$ edges and volume at most $N$.) We may assume, without loss of generality, that no edge of $G$ contains any point of the $X \times Y \times Z$ grid in its interior; any such edge can be replaced with a shorter edge without introducing any additional crossings. This modification may change the underlying graph, but does not change $m$ or $N$, which are the only quantities considered in the statement of the theorem. This assumption is subtle, but important, and is equivalent to assuming that, for every edge $u w$ of $G, \operatorname{gcd}\left(u_{1}-w_{1}, u_{2}-w_{2}, u_{3}-w_{3}\right)=1$.

For any integer, $p \geqslant 1$, define the $X \times Y \times Z$ p-grid as the set of points

$\{(x / p, y / p, z / p): x \in\{p, p+1, \ldots, p X\}, y \in\{p, p+1, \ldots, p Y\}, z \in\{p, p+1, \ldots, p Z\}\}$,

which we denote by $\mathbb{N}(p X, p Y, p Z) / p$. Observe that the size of the $p$-grid is at most $N p^{3}$. 


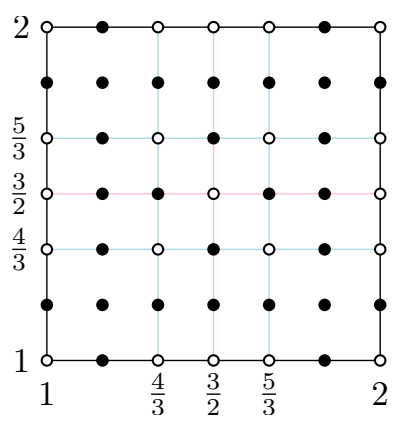

Figure 1: A 2-dimensional piece of the essential 6-grid. Removing the 1-grid, 2-grid, and 3 -grid from the 6 -grid leaves the essential 6-grid.

Notice that the $p$-grid and $q$-grid, for $q \neq p$ are not necessarily disjoint since, for example, the 4-grid includes the 2-grid. We wish to count crossings in $G$ by counting the number of crossings that occur on the $p$-grid for different values of $p$. However, in order to do this without double-counting any crossings we need a sequence of disjoint grids. To obtain these, we define the essential $p$-grid by starting with the $p$-grid and removing the $q$-grid for all $q \in\{1, \ldots, p-1\}$ such that $q$ divides $p$. (See Figure 1.)

Next, observe that each edge $u w$ of $G$ contains the $p$-grid points

$$
P_{u w}^{p}=\{u+(i / p)(w-u): i \in\{1, \ldots, p-1\}\}
$$

including the subset

$$
Q_{u w}^{p}=\{u+(i / p)(w-u): i \in\{1, \ldots, p-1\} \text { and } \operatorname{gcd}(i, p)=1\} .
$$

We shall now argue, using the assumption that $\operatorname{gcd}\left(u_{1}-w_{1}, u_{2}-w_{2}, u_{3}-w_{3}\right)=1$, that $Q_{u w}^{p}$ contains only essential $p$-grid points, More specifically, the points in $Q_{u w}^{p}$ are clearly on the $p$-grid, so the only concern is that some of these points are on the $q$-grid for some $q<p$. To see why this is not possible, observe that, if some point in $Q_{u w}^{p}$ were on the $q$-grid, for some $q<p$, this would imply that

$$
((i / p) x,(i / p) y,(i / p) z)=(a / q, b / q, c / q)
$$

for some integers $x=w_{1}-u_{1}, y=w_{2}-u_{2}, z=w_{3}-u_{3}, a, b$, and $c$ such that $\operatorname{gcd}(i, p)=1$ and $\operatorname{gcd}(x, y, z)=1$. Rewriting this gives

$$
(x, y, z)=(p a /(i q), p b /(i q), p c /(i q)) .
$$

Each value on the right hand side has a factor of $p$ in the numerator, so they must also have a factor of $p$ in the denominator. Otherwise, $\operatorname{gcd}(p a /(i q), p b /(i q), p c /(i q))>1=$ $\operatorname{gcd}(x, y, z)$. But this is not possible since $\operatorname{gcd}(i, p)=1$ and $q<p, \operatorname{so} \operatorname{gcd}(i q, p) \leqslant q<p$.

The size of the set $Q_{u w}^{p}$ is a well-studied quantity and is given by the Euler totient function $\varphi(p)=p \prod_{q \mid p}(1-1 / q)$, where $q$ ranges over all primes that divide $p[17$, Section 5.5]. Therefore, the total number of incidences between points of the essential $p$-grid 
and edges of $G$ is at least $m \cdot \varphi(p)$. In understanding the calculations that follow, it is helpful to pretend that $\varphi(p) \geqslant c p$ for some constant $0<c<1$, though this is not strictly correct since, for some $p, \varphi(p) \in O(p / \log \log p)$.

Let $x_{1}, \ldots, x_{\ell}$ denote the essential $p$-grid points that are incident to at least one edge and let $R_{i}$, with $i \in\{1, \ldots, \ell\}$, denote the number of edges incident to $x_{i}$. Observe that there are $\left(\begin{array}{c}R_{i} \\ 2\end{array}\right)$ crossing pairs of edges that cross at $x_{i}$. From the preceding discussion, we have $\sum_{i=1}^{\ell} R_{i} \geqslant m \cdot \varphi(p)$. Therefore, the total contribution of crossings that occur on the essential $p$-grid to $\mathrm{cr}_{3}(G)$ is at least

$$
\sum_{i=1}^{\ell}\left(\begin{array}{c}
R_{i} \\
2
\end{array}\right) \geqslant \ell\left(\begin{array}{c}
m \cdot \varphi(p) / \ell \\
2
\end{array}\right) \in \Omega\left(\ell(m \cdot \varphi(p) / \ell)^{2}\right)=\Omega\left(\frac{m^{2} \varphi(p)^{2}}{p^{3} N}\right) .
$$

The first inequality is an application of Jensen's Inequality to the function $f(x)=x(x-$ $1) / 2$. Since $\ell \leqslant N p^{3},\left(\begin{array}{c}m \cdot \varphi(p) / \ell \\ 2\end{array}\right) \geqslant 0$ for $p \leqslant \sqrt[3]{m / N}$ and the second inequality holds for $p \leqslant \sqrt[3]{m / N}$.

To finish, we need the following number-theoretic result which follows from well-known theorems and whose proof is included, for completeness, in Appendix A.

Claim 1. $\sum_{i=1}^{k} \varphi(i)^{2} / i^{3} \in \Omega(\log k)$

Finally, we can finish by summing over $p$ and using Claim 1 to obtain:

$$
\begin{aligned}
\operatorname{cr}(G) & \geqslant \sum_{p=1}^{\lfloor\sqrt[3]{m / N}\rfloor} \Omega\left(\frac{m^{2} \varphi(p)^{2}}{p^{3} N}\right) \\
& =\Omega\left(m^{2} / N\right) \sum_{p=1}^{\lfloor\sqrt[3]{m / N}\rfloor} \varphi(p)^{2} / p^{3} \\
& =\Omega\left(m^{2} / N\right) \log (m / N),
\end{aligned}
$$

as required.

\subsection{The Upper Bound}

In this section, we prove the following result:

Theorem 2. For all $m \leqslant N^{2} / 4, \operatorname{cr}_{3}(N, m) \in O\left(\left(m^{2} / N\right) \log (m / N)\right)$.

The proof of Theorem 2 follows easily from the following lemma:

Lemma 2. There exists a 3-D grid drawing of the complete bipartite graph $K_{k^{2}, k^{2}}$ on the $k \times k \times 2$ grid with $O\left(k^{6} \log k\right)$ crossings.

Before proving Lemma 2, we first show how it implies Theorem 2: For simplicity, in what follows, assume that each of $\sqrt{N}, \sqrt{m / N}$, and $N / \sqrt{m}$ are integers. Apply Lemma 2, 


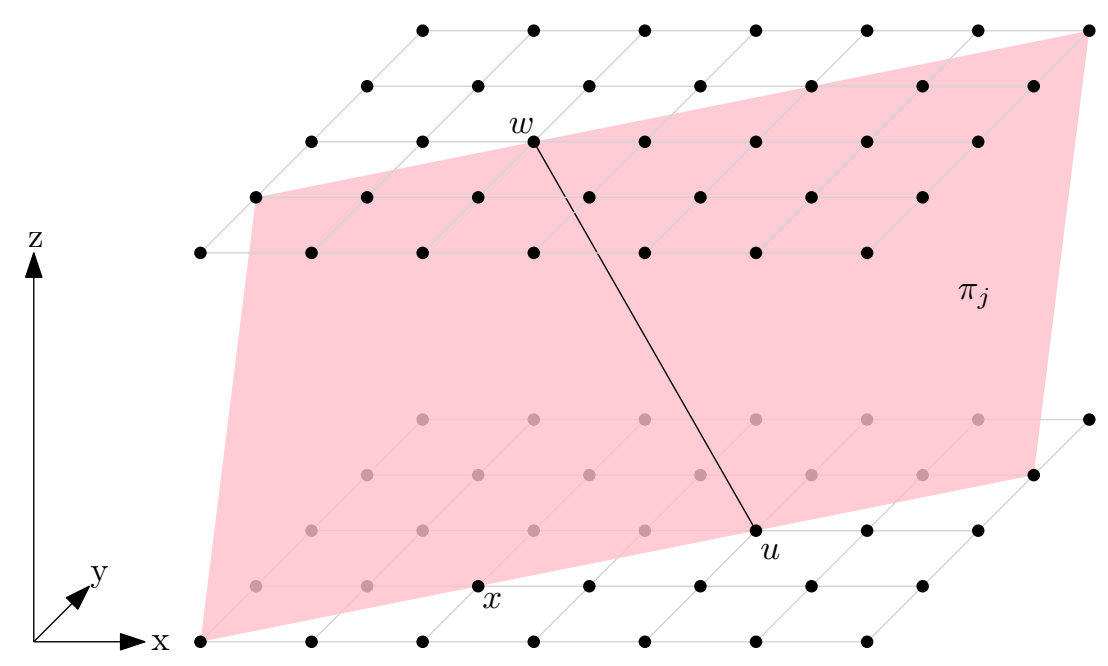

Figure 2: A plane $\pi_{j}$, defined by a point $x$, with $\operatorname{skip}\left(\pi_{j}\right)=2$. (z-coordinates are exaggerated.)

with $k=\sqrt{m / N}$ and tile the $\sqrt{N} \times \sqrt{N} \times 2$ grid with $N / k^{2}$ copies of this drawing. The resulting geometric graph has $2 N$ vertices, $m=N k^{4} / k^{2}=N k^{2}$ edges and

$$
O\left(\left(N / k^{2}\right) k^{6} \log k\right)=O\left(N k^{4} \log k\right)=O\left(\left(m^{2} / N\right) \log (m / N)\right)
$$

crossings, as required by Theorem 2 .

Proof of Lemma 2. The drawing of $K_{k^{2}, k^{2}}$ is the obvious one: each point of the $k \times k \times 2$ grid with z-coordinate 1 is connected by an edge to every point with z-coordinate 2 . We denote the resulting geometric graph by $G_{k^{2}}$.

We start by considering some edge $u w$ with $u_{3}=1$ (so $w_{3}=2$ ) and counting the number of edges that intersect $u w$. Let $\pi_{1}, \pi_{2}, \ldots$ be the planes that contain $u w$ and at least one additional vertex of $G$, whose z-coordinate is 1 . Observe that each such plane, $\pi_{j}$, contains a line in the plane $\left\{z \in \mathbb{R}^{3}: z_{3}=1\right\}$ and that this line contains $u$ and some vertex, $x$ such that $u x$ does not contain any other point of $\mathbb{Z}^{3}$ i.e., $\operatorname{gcd}\left(u_{1}-x_{1}, u_{2}-x_{2}\right)=1$ (see Figure 2). Define the skip of $\pi_{j}$ as

$$
\operatorname{skip}\left(\pi_{j}\right)=\max \left\{\left|u_{1}-x_{1}\right|,\left|u_{2}-x_{2}\right|\right\} .
$$

Observe that, if $\operatorname{skip}\left(\pi_{j}\right)=r$, then $\pi_{j}$ contains at most $2 k / r$ vertices of $G$ other than $u$ and $w$ (at most $k / r$ vertices from each "level" of $G$ ) and therefore contains at most $(k / r)^{2}$ edges that cross $u w$. Furthermore, the number of planes $\pi_{j}$ such that $\operatorname{skip}\left(\pi_{j}\right)=r$ is at most $4 r$ since each such plane is defined by two antipodal lattice points on the boundary of a square of side length $2 r$ centered at $u$; see Figure 3 . Therefore, the total number of edges that cross $u w$ is at most

$$
\sum_{r=1}^{k} 4 r(k / r)^{2}=4 k^{2} \sum_{r=1}^{k} 1 / r \leqslant 4 k^{2} \ln k+O\left(k^{2}\right) .
$$




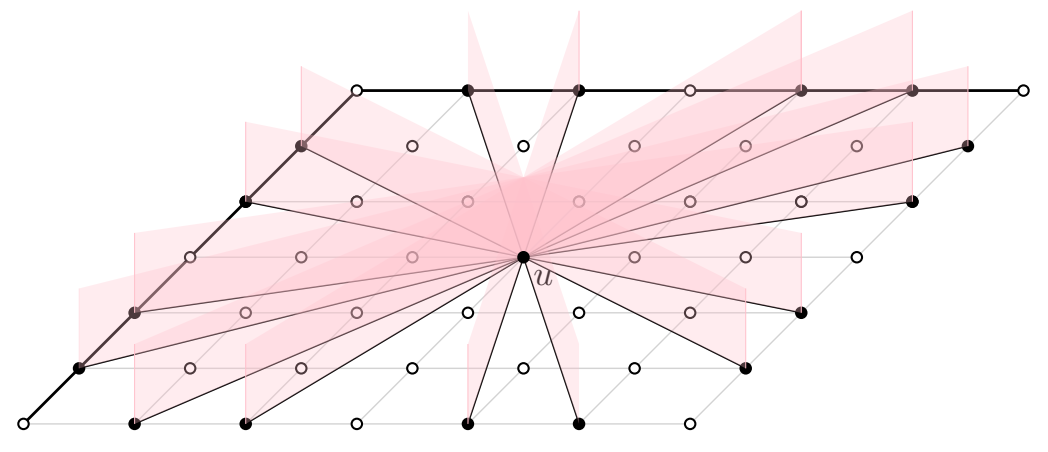

Figure 3: Each plane $\pi_{j}$ with $\operatorname{skip}\left(\pi_{j}\right)=r$ is defined by two antipodal lattice points on the boundary of a square of side length $2 r$ that is centered at $u$.

Since this is true for each of the $k^{4}$ edges, $u w$, we conclude that the total number of crossings in $G_{k^{2}}$ is at most $2 k^{6} \ln k+O\left(k^{6}\right) \in O\left(k^{6} \log k\right)$, as required.

\section{Higher Dimensions}

Next, we prove matching upper and lower bounds on $\mathrm{cr}_{d}(N, m)$ for $d \geqslant 4$.

\subsection{The Lower Bound}

Theorem 3. For all $m \geqslant 2^{d} N, \mathrm{cr}_{d}(N, m) \in \Omega\left(m^{2} / N\right)$. More specifically, $\operatorname{cr}_{d}(N, m) \geqslant$ $\frac{1}{2}\left(m^{2} /\left(2^{d}-1 N\right)-m\right)$, for all $m \geqslant 0$.

Proof. Let $G$ be any geometric grid graph with $m$ edges whose vertex set is contained in the $X_{1} \times \cdots \times X_{d}$ grid of volume at most $N$. As in the proof of Theorem 1 we may assume, without loss of generality, that no edge of $G$ contains a grid point. Observe that, for each edge $u w \in E(G)$, the midpoint $(u+w) / 2$ of $u w$ is contained in the $X_{1} \times \cdots \times X_{d}$ essential 2-grid:

$$
\begin{aligned}
& \mathbb{N}\left(2 X_{1}, \ldots, 2 X_{d}\right) / 2 \\
& \quad=\left\{\left(x_{1}, \ldots, x_{d}\right) / 2: x_{i} \in\left\{2,3, \ldots, 2 X_{i}\right\}, i \in\{1, \ldots, d\}\right\} \backslash \mathbb{N}\left(X_{1}, \ldots, X_{d}\right) .
\end{aligned}
$$

This 2-grid contains $K \leqslant\left(2^{d}-1\right) N$ points. Order the points of this 2-grid arbitrarily and let $R_{i}$ be the number of edges of $G$ whose midpoint is the $i$ th point of this 2 -grid. Then the number of crossings in $G$ is

$$
\operatorname{cr}(G) \geqslant \sum_{i: R_{i} \geqslant 1}\left(\begin{array}{c}
R_{i} \\
2
\end{array}\right)
$$




$$
\begin{aligned}
& =\frac{1}{2}\left(\sum_{i: R_{i} \geqslant 1}\left(R_{i}^{2}-R_{i}\right)\right) \\
& =\frac{1}{2}\left(\sum_{i: R_{i} \geqslant 1} R_{i}^{2}-m\right) \\
& \geqslant \frac{1}{2}\left(K(m / K)^{2}-m\right) \quad \text { (by Jensen's Inequality) } \\
& =\frac{1}{2}\left(m^{2} / K-m\right) \\
& \geqslant \frac{1}{2}\left(\frac{m^{2}}{\left(2^{d}-1\right) N}-m\right),
\end{aligned}
$$

as required.

\subsection{The Upper Bound}

Theorem 4. For all $d \geqslant 4$ and all $m \leqslant N^{2} / 4, \operatorname{cr}_{d}(N, m) \in O\left(m^{2} / N\right)$.

Proof. Let $\ell=k^{d-1}$ for some integer $k$. As in the proof of Theorem 2, it suffices to show that one can draw the complete bipartite graph $K_{\ell, \ell}$ on the $k \times \cdots \times k \times 2$ grid so that it has $O\left(k^{3(d-1)}\right)=O\left(\ell^{3}\right)$ crossings. Briefly, and omitting floors and ceilings: By taking $\ell=m / N$ (so that $\left.k=(m / N)^{1 /(d-1)}\right)$, we can tile the $N^{1 /(d-1)} \times \cdots \times N^{1 /(d-1)} \times 2$ grid with $\Theta(N / \ell)$ copies of $K_{\ell, \ell}$. The resulting drawing has $\Theta\left(\ell^{2} N / \ell\right)=\Theta(\ell N)=\Theta(m)$ edges and $\Theta\left(\ell^{3} N / \ell\right)=\Theta\left(m^{2} / N\right)$ crossings.

The remainder of this proof has the same structure as the proof of Lemma 2. The drawing of $K_{\ell, \ell}$ we use is the graph $G$ with $V(G)=\mathbb{N}(k, \ldots, k, 2)$ and

$$
\mathrm{E}(G)=\left\{u w \in V(G)^{2}: u_{d}=1 \text { and } w_{d}=2\right\} .
$$

Consider some edge $u w$ of $G$ with $u_{d}=1$ and $w_{d}=2$. Our strategy is to upper bound the number of edges that cross $u w$. Any edge $x y$ that crosses $u w$ is contained in some

plane, $\pi$, that contains $u w$ and $x y$. Without loss of generality, assume $x_{d}=1$. Let $x^{\prime}$ be some point of the integer lattice $\mathbb{Z}^{d}$ that is on the line containing $u x$ and such that $u x^{\prime}$ contains no point of $\mathbb{Z}^{d}$. (That is, $\operatorname{gcd}\left(u_{1}-x_{1}^{\prime}, \ldots, u_{d-1}-x_{d-1}^{\prime}\right)=1$.)

The plane $\pi$ that contains $u w$ and $x y$ can be expressed as

$$
\pi=\left\{u+t(w-u)+s\left(x^{\prime}-u\right): s, t \in \mathbb{R}\right\} .
$$

Restricted to the subspace $S_{u}=\left\{z \in \mathbb{R}^{d}: z_{d}=1\right\}, \pi$ becomes a line

$$
L_{u}=\pi \cap S_{u}=\left\{u+s\left(x^{\prime}-u\right): s \in \mathbb{R}\right\} .
$$

Similarly, restricted to the subspace $S_{w}=\left\{z \in \mathbb{R}^{d}: z_{d}=2\right\}, \pi$ becomes the parallel line:

$$
L_{w}=\pi \cap S_{w}=\left\{w+s\left(x^{\prime}-u\right): s \in \mathbb{R}\right\} .
$$


Observe that, since there is no point on the segment $u x^{\prime}$, the only points of the integer lattice $\mathbb{Z}^{d}$ contained in $L_{u}$ are obtained when the parameter $s$ is an integer:

$$
L_{u} \cap \mathbb{Z}^{d}=\left\{u+s\left(x^{\prime}-u\right): s \in \mathbb{Z}\right\}
$$

and, similarly,

$$
L_{w} \cap \mathbb{Z}^{d}=\left\{w+s\left(x^{\prime}-u\right): s \in \mathbb{Z}\right\} .
$$

If we define $r=\max \left\{\left|x_{i}^{\prime}-u_{i}\right|: i \in\{1, \ldots, d-1\}\right\}$, then we see that the number of vertices of $G$, other than $u$ and $w$, contained in each of $L_{u}$ and $L_{w}$ is at most $k / r$ (since $G$ 's vertices are contained in a box whose longest side has length $k$ ). Then, we define the skip of the plane $\pi$ to be $r$.

Now, consider all the planes that contain $u w$ and some other vertex of $G$. We wish to determine the number of such planes with skip $r$. Each such plane is defined by two antipodal grid points on the boundary of a $(d-1)$-hypercube of side length $2 r$, centered at $u$, that is contained in the $(d-1)$-dimensional subspace $\left\{z \in \mathbb{R}^{d}: z_{d}=1\right\}$. This hypercube has $2(d-1)$ facets and each facet contains $(2 r+1)^{d-2}$ grid points. Therefore, the total number of planes with skip $r$ is at most

$$
(d-1)(2 r+1)^{d-2}=(d-1)\left((2 r)^{d-2}+O\left(r^{d-3}\right)\right)
$$

Each plane with skip $r$ contains at most $(k / r)^{2}$ edges that cross $u w$. Therefore, the number, $X_{u w}$, of edges that cross $u w$ is at most

$$
\begin{aligned}
X_{u w} & \leqslant \sum_{r=1}^{k}(d-1)\left((2 r)^{d-2}+O\left(r^{d-3}\right)\right)(k / r)^{2} \\
& =(d-1) 2^{d-2} k^{2} \sum_{r=1}^{k}\left(r^{d-4}+O\left(r^{d-5}\right)\right) \\
& \leqslant(d-1) 2^{d-2} k^{2}\left(\frac{k^{d-3}}{d-3}+O\left(k^{d-4} \log k\right)\right) \quad(\text { since } d \geqslant 4) \\
& =\frac{(d-1) 2^{d-2} k^{d-1}}{d-3}+O\left(k^{d-2} \log k\right) \\
& =O\left(k^{d-1}\right)=O(\ell) .
\end{aligned}
$$

Since there are $\ell^{2}$ edges, the total number of crossings is therefore $O\left(\ell^{3}\right)$, as required.

\section{The Number of Non-Crossing Graphs}

In this section, we show that, for dimensions $d \geqslant 4, \operatorname{ncs}_{d}(N) \in 2^{\Theta(N \log N)}$, i.e., the maximum number of crossing-free graphs that can be drawn on any grid of volume $N$ is $2^{\Theta(N \log N)}$.

The upper bound follows easily from Lemma 1 which states that any crossing-free $d$-D geometric grid graph of volume $N$ has at most $\left(2^{d}-1\right) N$ edges. Therefore, any such 
graph is a subgraph of a graph obtained by choosing at most $\left(2^{d}-1\right) N$ edges from among the at most $\left(\begin{array}{c}N \\ 2\end{array}\right)$ possible edges. Each graph with $\left(2^{d}-1\right) N$ edges has $2^{\left(2^{d}-1\right) N}$ subsets of edges. Therefore, the number of such graphs is at most

$$
\begin{aligned}
\operatorname{ncs}_{d}(N) \leqslant 2^{\left(2^{d}-1\right) N}\left(\begin{array}{c}
\left(\begin{array}{c}
N \\
2
\end{array}\right) \\
\left(2^{d}-1\right) N
\end{array}\right) & \leqslant 2^{\left(2^{d}-1\right) N}\left(N^{2}\right)^{\left(2^{d}-1\right) N} \\
& =2^{2\left(2^{d}-1\right) N \log N+\left(2^{d}-1\right) N} \in 2^{O(N \log N)} .
\end{aligned}
$$

The preceding argument is standard and is used, for example, by Bárat et al. [4, Lemma 4] for upper-bounding the maximum number of crossing-free plane graphs with $m$ edges that can be drawn on any particular set of $n$ points in the plane.

Next we show that the number of crossing-free geometric graphs that can be drawn on the $N^{1 /(d-1)} \times \cdots \times N^{1 /(d-1)} \times 2$ grid is at least $2^{\Omega(N \log N)}$.

Theorem 5. For all $d \geqslant 4, \operatorname{ncs}_{d}(N) \in 2^{\Omega(N \log N)}$.

Proof. Let $G_{N}$ denote the complete bipartite geometric graph described in the proof of Theorem 4 with the value $\ell=N / 2$ (assuming, only for simplicity, that $(N / 2)^{1 /(d-1)}$ is an integer). For a geometric graph, $G$, let $\operatorname{ncs}(G)$ denote the number of crossing-free subgraphs of $G$ that have the same vertex set as $G$ by with a subset of $G$ 's edges. Observe that $\operatorname{ncs}(G) \geqslant 1$ since the subgraph obtained by removing all edges from $G$ is crossing-free. Now define

$$
f(m)=\min \left\{\operatorname{ncs}(G): G \text { is a subgraph of } G_{N} \text { having } m \text { edges }\right\} .
$$

Our goal is to lower-bound $f\left(N^{2}\right)$. In order to do this, we establish a recurrence inequality and base cases.

For our base cases, we have

$$
f(m) \geqslant 1
$$

for all $m \geqslant 0$, since $\operatorname{ncs}(G) \geqslant 1$ for all graphs, $G$.

Let $c=c(N) \in O(1)$ be a function such that $c N$ is an integer and, for all sufficiently large $N$, every edge of $G_{N}$ intersects at most $c N-1$ other edges. By the proof of Theorem 4 such a function $c$ exists. Fix any subgraph, $G$, of $G_{N}$ that has $m \geqslant c N$ edges. From $G$, select any edge $e$. Then there are at least $f(m-1)$ crossing-free subgraphs of $G$ that do not include $e$. Furthermore, $e$ intersects at most $c N-1$ other edges of $G$, so there are at least $f(m-c N)$ crossing-free subgraphs of $G$ that include $e$. Therefore,

$$
f(m) \geqslant f(m-1)+f(m-c N)
$$

for $m \geqslant c N$. Repeatedly expanding the first term gives:

$$
\begin{aligned}
f(m) & \geqslant f(m-1)+f(m-c N) \\
& \geqslant f(m-1)+f(m-2 c N) \\
& \geqslant f(m-2)+2 f(m-2 c N)
\end{aligned}
$$




$$
\begin{aligned}
& \geqslant f(m-3)+3 f(m-2 c N) \\
& \vdots \\
& \geqslant c N \times f(m-2 c N)
\end{aligned}
$$

as long as $m \geqslant 2 c N$.

For an integer $t$, we can iterate (4) $t$ times to obtain

$$
f(m) \geqslant(c N)^{t} \times f(m-2 c t N),
$$

for $m \geqslant 2 c t N$. Taking $m=N^{2}$, (5) becomes

$$
f\left(N^{2}\right) \geqslant(c N)^{t} \times f\left(N^{2}-2 c t N\right) \geqslant(c N)^{t},
$$

for $t \leqslant N /(2 c)$. Taking $t=\lfloor N /(2 c)\rfloor$ then yields the desired result:

$$
f\left(N^{2}\right) \geqslant(c N)^{\lfloor N /(2 c)\rfloor} \geqslant(c N)^{N /(2 c)-1}=2^{\left(\frac{N}{2 c}-1\right)(\log N+\log c)} \in 2^{\Omega(N \log N)} .
$$

We remark that the proof of Theorem 5 also works to lower-bound the number of crossing-free matchings in $G_{N}$. When one selects an edge $u w$ to be part of the matching, the at most $c N$ edges of $G_{N}$ that intersect $u w$ and the $2 N-1$ edges that have $u$ or $w$ as an endpoint must be discarded. Thus, one discards at most $(c+2) N$ edges and the remainder of the proof goes through unmodified.

Corollary 1. For all $d \geqslant 4$ and $N=2 k^{d-1}$, the number of crossing-free matchings with vertex set $\mathbb{N}(k, \ldots, k, 2)$ is $2^{\Omega(N \log N)}$.

From Corollary 1, we can derive a lower-bound on the number of crossing-free spanning trees of the $k \times \cdots \times k \times 2$ grid:

Corollary 2. For all $d \geqslant 4$ and $N=2 k^{d-1}$. The number of crossing-free trees with vertex set $\mathbb{N}(k, \ldots, k, 2)$ is $2^{\Omega(N \log N)}$.

Proof. Each of the crossing-free matchings counted by Corollary 1 uses only edges uw of $G_{N}$ such that $u_{d}=1$ and $w_{d}=2$. Each such matching, $M$, can be augmented into a crossing-free connected graph, $G_{M}$, with vertex set $\mathbb{N}(k, \ldots, k, 2)$ by, for example, adding all edges in the set

$$
\left\{u w: u, w \in \mathbb{N}(k, \ldots, k, 2), u_{d}=w_{d} \text { and }\|u-w\|=1\right\} .
$$

(The edges in the preceding set are those of two finite $(d-1)$-dimensional lattices, one in the subspace $\left\{x \in \mathbb{R}^{d}: x_{d}=1\right\}$ and the other in the subspace $\left\{x \in \mathbb{R}^{d}: x_{d}=2\right\}$.) The graph $G_{M}$ can be reduced to a tree, $T_{M}$, that includes all edges of $M$ by repeatedly finding a cycle, $C$, and removing any edge of $C$ that is not part of $M$. (An edge of $C \backslash M$ exists because $M$ is a matching, and hence acyclic.) After each such modification, $G_{M}$ remains connected and has fewer cycles. This processes terminates when $G_{M}$ becomes the desired tree $T_{M}$.

Thus, for each of the $2^{\Omega(N \log N)}$ matchings, $M$, there exists a spanning tree $T_{M}$ that contains $M$. Any spanning tree with $N$ vertices contains no more than $2^{N-1}$ matchings and therefore, there are at least $2^{\Omega(N \log N)} / 2^{N-1} \in 2^{\Omega(N \log N)}$ crossing-free spanning trees with vertex set $\mathbb{N}(k, \ldots, k, 2)$. 
We finish this section by observing that our lower bounds are not just for "flat" grids like the $k \times \cdots \times k \times 2$ grid. They also hold for the "square" $k \times \cdots \times k$ grid.

Corollary 3. For all $d \geqslant 4$ and $N=k^{d}$, the number of crossing-free matchings and spanning trees with vertex set $\mathbb{N}(k, \ldots, k)$ is $2^{\Omega(N \log N)}$

Proof. Observe that the $k \times \cdots \times k$ grid is made up of $k$ layers, each of which is a $k \times \cdots \times k \times 1$ grid. Between any consecutive pair of these layers there are, by Corollary 1 , $2^{\Omega\left(k^{d-1} \log k\right)}$ crossing-free matchings that contain only edges that span both layers. Since there are $k-1$ consecutive pairs of layers, there are therefore

$$
\left(2^{\Omega\left(k^{d-1} \log k\right)}\right)^{k-1}=2^{\Omega\left(k^{d} \log k\right)}=2^{\Omega(N \log N)}
$$

crossing-frees graphs whose vertex set is the $k \times \cdots \times k$ grid.

Note that the graphs we obtain in the preceding manner contain no cycles. Therefore, to obtain a lower-bound of $2^{\Omega(N \log N)}$ on the number of spanning trees we can augment any of these graphs into a crossing-free spanning tree as is done in the proof of Corollary 2 .

To obtain a lower-bound on the number of matchings we can simply count the matchings that only include edges from layer $i$ to layer $i+1$ with $i \equiv 1(\bmod 2)$. There are

$$
\left(2^{\Omega\left(k^{d-1} \log k\right)}\right)^{\lfloor(k-1) / 2\rfloor}=2^{\Omega\left(k^{d} \log k\right)}=2^{\Omega(N \log N)}
$$

such matchings.

\section{$5 \quad$ Summary and Remarks}

We have given matching upper and lower bounds on the minimum number of crossings in $d$-D geometric grid graphs with $m$ edges and volume at most $N$. The upper-bound $\mathrm{cr}_{d}(N, m) \in O\left(m^{2} / N\right)$, for $d \geqslant 4$, allows the application of a recursive counting technique to show the lower-bound $\operatorname{ncs}_{d}(N) \in 2^{\Omega(N \log N)}$; this is similar to way in which Ajtai et al. [3] used the lower-bound $\operatorname{cr}(n, m) \in \Omega\left(m^{3} / n^{2}\right)$ to show that that the maximum number of planar graphs that can be drawn on any point set of size $n$ is $2^{O(n)}$. This $2^{\Omega(N \log N)}$ lower-bound also holds if we restrict the graphs to be spanning trees or matchings, but we know very little about spanning cycles:

Open Problem 1. Determine the maximum number of crossing-free spanning cycles whose vertex set is a grid of volume $N$.

The natural open problem left in this area is that of finding tight bounds on $\operatorname{ncs}_{3}(N)$. A lower bound of $2^{\Omega(n)}$ follows immediately from the fact that there exists a non-crossing 3-D grid graph with volume $N$ and $\Omega(N)$ edges (so this graph has $2^{\Omega(n)}$ non-crossing subgraphs). An upper bound of $2^{O(N \log N)}$ can be obtained using the argument given at the beginning of Section 4.

Open Problem 2. Find non-trivial bounds $-2^{o(N \log N)}$ or $2^{\omega(N)}-$ on $\operatorname{ncs}_{3}(N)$. 
Open Problem 2, which was the original motivation for the current work, was communicated to the first author by David R. Wood and his motivation for asking this question comes from a question of Pach et al. [23], who ask "Does every graph with $n$ vertices and maximum degree three have a crossing-free 3-D grid drawing of volume $O(n)$ ?" This question remains unresolved, even when the maximum degree three condition is relaxed to maximum degree $O(1)$.

If Open Problem 2 can be answered with a non-trivial upper bound, then this would settle Pach et al.'s question as follows. The number of labelled graphs with $n$ vertices and having maximum degree 3 is $2^{(3 / 2) n \log n-O(n)}[4$, Appendix A]. On the other hand, if one can show that $\operatorname{ncs}_{3}(N) \in 2^{o(N \log N)}$, then for every constant $c>0$,

$$
n ! \operatorname{ncs}_{3}(c n)=n ! 2^{o(n \log n)} \leqslant 2^{n \log n+o(n \log n)}<2^{(3 / 2) n \log n-O(n)},
$$

for sufficiently large $n$. This would answer Pach et al.'s question in the negative, since there would be more labelled $n$-vertex graphs of maximum degree three than there are labelled 3-D geometric grid graphs of volume $c$ for any constant $c$. This type of counting argument has been used successfully to answer similar questions about geometric thickness [4], distinct distances [8], slope number [21], book thickness [20], and queue number $[20,31]$.

Another approach to resolving the question of Pach et al. is to consider that there are maximum degree 3 graphs that have some properties that would seem to rule out a linear volume embedding. An obvious candidate property is that of being an expander: There exist graphs, $G$, with maximum degree $O(1)$ and such that, for any subset $S \subseteq V(G)$, $|S| \leqslant n / 2$ the number of vertices of $V(G) \backslash S$ adjacent to at least one vertex in $S$ is at least $\epsilon|S|$, for some constant $\epsilon>0$.

Expanders have no separator of size $o(n)$ and are therefore non-planar [19]. However, very recently Bourgain and Yehudayoff [6] have shown that there exist bounded degree graphs that are expanders and that have constant queue number. Through a result of Dujmović et al. [13, Theorem 8], this implies that there are constant degree expanders that can be drawn on a 3-dimensional grid with volume $O(n)$. Thus, the property of expansion is not sufficient to rule out linear volume $3-\mathrm{D}$ grid drawings. We are still no closer to solving Pach et al.'s 14 year old problem:

Open Problem 3 (Pach et al. 1999). Does every graph with $n$ vertices and maximum degree three have a crossing-free $3-\mathrm{D}$ grid drawing of volume $O(n)$ ?

\section{Acknowledgements}

This research was initiated at the AMS Mathematics Research Communities Workshop on Discrete and Computational Geometry, June 10-16, 2012. The work of Vida Dujmović and Pat Morin was partly funded by NSERC. Adam Sheffer was partially supported by Grant 338/09 from the Israel Science Fund and by the Israeli Centers of Research Excellence program (Center No. 4/11). 


\section{References}

[1] E. Ackerman. On topological graphs with at most four crossings per edge. Manuscript, 2013.

[2] M. Aigner and G. M. Ziegler. Proofs from THE BOOK. Springer, 1999.

[3] M. Ajtai, V. Chvátal, M. M. Newborn, and E. Szemerédi. Crossing-free subgraphs. Annals of Discrete Mathematics, 12:9-12, 1982.

[4] J. Bárat, J. Matoušek, and D. R. Wood. Bounded-degree graphs have arbitrarily large geometric thickness. Electronic Journal of Combinatorics, 13:\#R3:1-14, 2006.

[5] P. Bose, J. Czyzowicz, P. Morin, and D. R. Wood. The maximum number of edges in a three-dimensional grid-drawing. Journal of Graph Algorithms and Applications, 8(1):21-26, 2004.

[6] J. Bourgain and A. Yehudayoff. Expansion in $\mathrm{SL}_{2}(\mathbb{R})$ and monotone expansion. Geometric and Functional Analysis, 23(1):1-41, 2013.

[7] B. Bukh and A. Hubard. Space crossing numbers. Combinatorics, Probability and Computing, 21(3):358-373, 2012.

[8] P. Carmi, V. Dujmović, P. Morin, and D. R. Wood. Distinct distances in graph drawings. Electronic Journal of Combinatorics, 15:\#R107:1-23, 2008.

[9] R. F. Cohen, P. Eades, T. Lin, and F. Ruskey. Three-dimensional graph drawing. Algorithmica, 17(2):199-208, 1996.

[10] T. K. Dey. Improved bounds on planar $k$-sets and related problems. Discrete $\mathscr{G}$ Computational Geometry, 19:373-382, 1998.

[11] G. Di Battista, F. Frati, and J. Pach. On the queue number of planar graphs. SIAM Journal on Computing, 42(6):2243-2285, 2013.

[12] V. Dujmović, P. Morin, and D. R. Wood. Layout of graphs with bounded tree-width. SIAM Journal on Computing, 34(3):553-579, 2005.

[13] V. Dujmović, A. Pór, and D. R. Wood. Track layouts of graphs,. Discrete Mathematics and Theoretical Computer Science, 6(2):497-522, 2004.

[14] V. Dujmović and D. R. Wood. Three-dimensional grid drawings with sub-quadratic volume. In János Pach, editor, Towards a Theory of Geometric Graphs, volume 342 of Contemporary Mathematics, pages 55-66. AMS, 2004.

[15] V. Dujmović and D. R. Wood. Upward three-dimensional grid drawings of graphs. Order, 23(1):1-20, 2006.

[16] S. Felsner, G. Liotta, and S. Wismath. Straight-line drawings on restricted integer grids in two and three dimensions. Journal of Graph Algorithms and Applications, 7(4):363-398, 2003.

[17] G. H. Hardy and E. M. Wright. An Introduction to the Theory of Numbers. Oxford Science Publications. Oxford University Press, 1979. 
[18] M. Hoffmann, M. Sharir, A. Sheffer, C. D. Tóth, and E. Welzl. Counting plane graphs: Flippability and its applications. In Frank Dehne, John Iacono, and JörgRüdiger Sack, editors, Proceedings of the 12th Symposium on Algorithms and Data Structures (WADS 2011), volume 6844 of Lecture Notes in Computer Science, pages 524-535. Springer, 2011.

[19] R. J. Lipton and R. E. Tarjan. A separator theorem for planar graphs. SIAM Journal on Applied Mathematics, 36:177-189, 1979.

[20] S. M. Malitz. Graphs with $E$ edges have pagenumber $O(\sqrt{E})$. Journal of Algorithms, 17(1):71-84, 1994.

[21] J. Pach and D. Pálvölgyi. Bounded-degree graphs can have arbitrarily large slope numbers. Electronic Journal of Combinatorics, 13:\#N1:1-4, 2006.

[22] J. Pach, R. Radoičić, G. Tardos, and G. Tóth. Improving the crossing lemma by finding more crossings in sparse graphs. Discrete \& Computational Geometry, 36(4):527552, 2006.

[23] J. Pach, T. Thiele, and G. Tóth. Three-dimensional grid drawings of graphs. In Bernard Chazelle, Jacob E. Goodman, and Richard Pollack, editors, Advances in discrete and computational geometry, volume 223 of Contemporary Mathematics, pages 251-255. AMS, 1999.

[24] M. Sharir and A. Sheffer. Counting triangulations of planar point sets. Electronic Journal of Combinatorics, 18(1):P70:1-74, 2011.

[25] M. Sharir and A. Sheffer. Counting plane graphs: Cross-graph charging schemes. Combinatorics, Probability and Computing, 22(6):935-954, 2013.

[26] M. Sharir, A. Sheffer, and E. Welzl. Counting plane graphs: perfect matchings, spanning cycles, and Kasteleyn's technique. Journal of Combinatorial Theory, Series A, 120:777-794, 2013.

[27] M. Sharir and E. Welzl. On the number of crossing-free matchings, cycles, and partitions. SIAM Journal on Computing, 36(3):695-720, 2006.

[28] A. Sheffer. Numbers of plane graphs. Available from: http://www.cs.tau.ac.il/ sheffera/counting/PlaneGraphs.html [cited 2012-12-10].

[29] L. A. Székely. Crossing numbers and hard Erdös problems in discrete geometry. Combinatorics, Probability and Computing, 6(3):353-358, 1997.

[30] E. Szemerédi and W. Trotter. Extremal problems in discrete geometry. Combinatorica, 3:381-392, 1983.

[31] David R. Wood. Bounded-degree graphs have arbitrarily large queue-number. Discrete Mathematics \& Theoretical Computer Science, 10(1):27-34, 2008. 


\section{A Proof of Claim 1}

Proof of Claim 1. We must prove that $\sum_{i=1}^{k} \phi(i)^{2} \in \Omega(\log k)$. Recall the following result on Euler's totient function [17, Theorem 330]:

$$
\left(3 / \pi^{2}\right) n^{2}-O(n) \leqslant \sum_{i=1}^{n} \varphi(i) \leqslant\left(3 / \pi^{2}\right) n^{2}+O(n \log n) .^{2}
$$

Using this result and the Cauchy-Schwartz Inequality, we obtain

$$
\left(3 / \pi^{2}\right) n^{2}-O(n) \leqslant \sum_{i=1}^{n} \varphi(i)=\sum_{i=1}^{n} \varphi(i) \cdot 1 \leqslant \sqrt{\sum_{i=1}^{n} \varphi(i)^{2}} \cdot \sqrt{\sum_{i=1}^{n} 1}=\sqrt{\sum_{i=1}^{n} \varphi(i)^{2}} \cdot \sqrt{n} .
$$

Dividing by $\sqrt{n}$ and squaring, we obtain

$$
\sum_{i=1}^{n} \varphi(i)^{2} \geqslant\left(9 / \pi^{4}\right) n^{3}-O\left(n^{2}\right)+O(n) \geqslant\left(9 / \pi^{4}\right) n^{3} \geqslant n^{3} / 11
$$

for all $n \geqslant N_{0}$, for some sufficiently large $N_{0}$. On the other hand, $\varphi(i)<i$, so

$$
\sum_{i=1}^{n} \varphi(i)^{2}<\sum_{i=1}^{n} i^{2} \leqslant \sum_{i=1}^{n} n^{2}=n^{3}
$$

We can now prove the claim as follows:

$$
\begin{aligned}
\sum_{i=1}^{k} \varphi(i)^{2} / i^{3} \geqslant \sum_{i=N_{0}}^{k} \varphi(i)^{2} / i^{3} & \geqslant \sum_{j=N_{0}}^{\left\lfloor\log _{3} k\right\rfloor} \sum_{i=3^{j-1}+1}^{3^{j}} \varphi(i)^{2} / i^{3} \\
& \geqslant \sum_{j=N_{0}}^{\left\lfloor\log _{3} k\right\rfloor} \frac{1}{3^{3 j}}\left(\sum_{i=3^{j-1}+1}^{3^{j}} \varphi(i)^{2}\right) \\
& =\sum_{j=N_{0}}^{\left\lfloor\log _{3} k\right\rfloor} \frac{1}{3^{3 j}}\left(\sum_{i=1}^{3^{j}} \varphi(i)^{2}-\sum_{i=1}^{3^{j-1}} \varphi(i)^{2}\right) \\
& \geqslant \sum_{j=N_{0}}^{\left\lfloor\log _{3} k\right\rfloor} \frac{1}{3^{3 j}}\left(3^{3 j} / 11-\left(3^{j-1}\right)^{3}\right) \\
& \geqslant \sum_{j=N_{0}}^{\left\lfloor\log _{3} k\right\rfloor} \frac{1}{3^{3 j}}\left(3^{3 j} / 11-3^{3 j} / 27\right) \\
& =\sum_{j=N_{0}}^{\left\lfloor\log _{3} k\right\rfloor} \Omega(1)=\Omega(\log k) . \square
\end{aligned}
$$

\footnotetext{
${ }^{2}$ Hardy and Wright state the result as $\sum_{i=1}^{n} \varphi(i)=\left(3 / \pi^{2}\right) n^{2}+O(n \log n)$. However, in their notation $f(n)=O(g(n))$ means $|f(n)| \leqslant c g(n)$ for some constant $c>0$. This leads to the statement given here.
} 